Diánoia, vol. 29, no. 29, 1983

\title{
SUSTANCIA MATERIAL EN LA CORRESPONDENCIA CON ARNAULD
}

\author{
ENRIQUe VillanUeVA
}

INSTITUTO DE INVESTIGaciones Filosbficas Universidad Nacional Autónoma de México

Uno de los aspectos cruciales de la teoría de la sustancia de Leibniz lo constituye su teoría de la sustancia material individual. Aun cuando hay pasajes difíciles en los escritos de 1686, se puede afirmar que Leibniz acepta que los cuerpos materiales alcancen la calificación de sustancias, es decir, de entidades dotadas con unidad verdadera.

En el presente trabajo me propongo examinar la tesis de Leibniz tomando como base su respuesta a Arnauld al final de la correspondencia que ambos sostuvieron. Mi interés consiste en determinar en este estadio hasta qué punto está Leibniz decidido a admitir sustancias materiales. Es decir, sustancias que no sean espirituales. La respuesta a esa pregunta implica las respuestas a otras preguntas como son: en qué consiste la unidad de una sustancia material?, ¿cuáles son sus constituyentes y cómo se unifican?, ¿cómo preserva su identidad en el tiempo la unidad así constituida? Finalmente, hay otras cuestiones, lingüísticas y epistemológicas, que deben plantearse, como por ejemplo: ¿cómo deben ser los nombres de las sustancias materiales?, ¿cuánto conocemos de las sustancias materiales y cuánto podemos conocer de las mismas?

En la primera parte de este trabajo interpreto las objeciones de $\mathrm{Ar}$ nauld. En la segunda propongo una interpretación variada de las respuestas de Leibniz. Finalmente, en la tercera parte presento unas conclusiones escépticas de las tesis de Leibniz en este periodo.

La prolongada correspondencia entre Arnauld y Leibniz llega a un climax con la carta que le envía Arnauld a Leibniz el 28 de agosto de 1687 (G. II, pp. 105-109) ${ }^{1}$ y en la cual Arnauld, después de las numerosas

1 Las citas corresponden a la edición de Gerhardt y se indica el volumen y la página. Mi numeración latina corresponde, debidamente ajustada, a la numeración que hace Arnauld y que Leibniz sigue en su carta de respuesta. La carta de Arnauld 
cuanto repetidas afirmaciones de Leibniz, vuelve a exponer sus problemas acerca de la tesis de Leibniz sobre los individuos sustanciales. ${ }^{2}$ Arnauld expresa en su carta tres objeciones fundamentales, la última de las cuales se ramifica en seis. Las resumiré numerándolas según lo antes expuesto.

1. La primera concierne a la tesis de la expresión y toca al problema de la mente y el cuerpo. ${ }^{3}$

2. La segunda versa sobre el movimiento de las sustancias corporales y toca los problemas de la existencia de Dios, de la mente y el cuerpo y de la libertad.4

3. La tercera es acerca de las formas sustanciales que siendo indivisibles e indestructibles confieren unidad a la materia (misma que se concibe como divisible al infinito). Esta objeción se subdivide en seis y de ellas me ocuparé a continuación.

3.1 Arnauld piensa que la materia es el más imperfecto de los seres porque no es unum ens sino siempre plura entia. Arnauld le pregunta a Leibniz por qué no puede haber plura entia sin unum ens, es decir, que haya cuerpos sin forma sustancial. ${ }^{\circ}$

3.2 Arnauld objeta que la forma sustancial puede conferir unidad a las partículas de materia y presenta el siguiente argumento:

i) El atributo unum debe ser esencial e intrínseco al unum ens.

ii) Las partículas de materia sólo son plura entia.

iii) La unidad de la materia le vendrá de una denominación extrínseca al imponerle la forma sustancial que le es extraña, nunca de una denominación intrínseca.

Las partículas de materia no son unum en sentido metafísico riguroso. ${ }^{6}$

3.3 Arnauld argumenta, en consecuencia, que la forma sustancial no puede eliminar la pluralidad. El argumento se puede reconstruir de la siguiente manera:

se encuentra en el Vol. II, pp. 105-109; la de Leibniz está en el mismo volumen, pp. 111-128:

2 Para un examen de las dificultades de la tesis de Leibniz acerca de las sustancias individuales en los escritos de 1686, véase mi artículo "Las tesis de la unidad sustancial en los escritos de Leibniz circa 1686, en Filosofia e Epistemologia, V, Lisboa. También en III Simposio Internacional de Filosofia, Ediciones del Instituto de Investigaciones Filosóficas, UNAM, México. En prensa.

3 G. II, pp. 105-106.

4 Ibid., p. 106 .

s Ibid., pp. 106-107.

6 Ibid., p. 107. 
Las formas sustanciales se admiten solamente en los cuerpos animados. Pero los cuerpos animados son organizados y son plura entia. Luego, las formas sustanciales no privan a los cuerpos animados de su pluralidad sino antes bien presuponen esta pluralidad para que se exija la unidad. ${ }^{7}$ En otras palabras, Arnauld objeta que se pueda interpretar la función unificadora de la forma sustancial bajo el modelo de la simplicidad.

3.4 Arnauld objeta que la unidad de los espíritus o mentes se puede lograr con el pensamiento. Su argumento se puede reconstruir así:

i) Sólo hay dos clases de sustancias, a saber, cuerpos o espíritus.

ii) Si son cuerpos, son extensas y divisibles al infinito.

iii) Pero entonces esos cuerpos serán plura entia sin unidad verdadera.

iv) Si son espíritus serán unum ens pero solamente si piensan.

v) Es difícil pensar que los gusanos o las ostras piensan.

Luego, las almas de los animales o bien carecen de unidad, o no son materiales. ${ }^{8}$

3.5 Arnauld objeta a la indestructibilidad de las almas de los animales de la siguiente manera:

i) La muerte de un animal no debe concebirse como destrucción de su alma o como transfiguración sino como transformación del mismo animal que guarda siempre la misma alma.

ii) Pero cuando se quema un gusano de seda no diremos que conserva la misma alma unida a una pequeña partícula de ceniza pues si así fuese, ¿por qué no nacen otros gusanos de esa partícula de ceniza? ${ }^{9}$

3.6 Arnauld objeta otro ejemplo acerca de la inmortalidad del alma de los animales.

¿Qué sucede con el Cordero que inmoló Abraham? ¿Permanece su alma en las cenizas? No parece poder componer un animal con ella según las leyes de la naturaleza. ¿También aquí la muerte es solamente transformación? 10

Las seis objeciones de Arnauld acerca de las formas sustanciales tocan diversos aspectos de éstas pero lo que las une a todas ellas se puede expresar diciendo que Leibniz ha dejado en una oscuridad fundamental la elucidación metafísica de la manera según la cual, las formas sustanciales cumplen la función de unificar una pluralidad en un solo ente indivisible $e$ indestructible. Para decirlo en una forma más intuitiva y plástica, Leibniz no descubre el mecanismo según el cual una diversidad

8 Ibid., pp. 107-108.

I Ibid., pp. 108-109.

10 Ibid., p. 109. 
ontológica se ve llevada a constituir una unidad indivisa e indestructible y con ello puede calificar como un individuo primario y principal en nuestro reino ontológico (según la reconstrucción racional que hace Leibniz del mismo).

Leibniz respondió a Arnauld el 9 de octubre de 1687. Dada la fecha de la carta podemos estar seguros de que su respuesta exhibe su idea definitiva de la sustancia individual en esta época - por lo menos- y por ello nos parece importante sintetizarla. Me concentraré en la respuesta de Leibniz a las seis objeciones de Arnauld respecto de la forma sustancial de las sustancias materiales.

En general, Leibniz reitera que las formas o almas son indivisibles e indestructibles. En lo particular, responde de la siguiente manera:

3.1 Leibniz reitera a priori que la multiplicidad supone unidad. Éste "supone" no lo aclara Leibniz y puede ser, como veremos después, una tesis de composición o una tesis de unificación. ${ }^{11}$

Luego rechaza que toda forma sustancial sea un alma, así como que los cuerpos animados sean pocos en número.

Finalmente establece que la materia está compuesta de esos cuerpos animados dotados de una entelequia primitiva o de un principio vital o sea de sustancias corpóreas.

En suma, la respuesta de Leibniz consiste en afirmar que concede que la materia es plura entia pero que se tiene que componer de una infinidad de sustancias corpóreas, esto es, de unidades pues de otra manera, parece ser, sería una nada.

No dice Leibniz aún qué sean esas sustancias corpóreas ni cuál su principio de unidad. Tampoco aclara cómo se condicen las unidades de las sustancias corpóreas componentes con la unidad de la sustancia de esa manera compuesta. El problema se complica pues ahora resulta que la forma sustancial no solamente debe unificar una pluralidad, sino que debe unificar una pluralidad de unidades.

3.2 Leibniz parece aceptar la premisa (i) pero rechaza la (ii) distinguiendo dos sentidos de materia; a saber, la materia que pertenece a la sustancia animada forma verdaderamente un ser y la materia considerada como masa es

11 G. II, p. 118. Otra manera de reconstruir el argumento de Leibniz consiste en afirmar que a menos que se le concedan las sustancias materiales se caerá en un regressus; pero para salvarse de tal absurdo Leibniz cae en una perplejidad mayor, según veremos más abajo. 
un puro fenómeno o apariencia bien fundada lo mismo que el espacio y el tiempo. (G. II, pp. 118-119).

$\mathbf{Y}$ pasa a formular un argumento sumamente fuerte de acuerdo con el cual ni la figura, ni la magnitud ni el movimiento ni ninguna otra cualidad puede dar cuenta o justificar la realidad metafísica de la materia y, en consecuencia, Leibniz equipara a la materia como masa a

un fenómeno mero y simple como el arco iris (ibid.).

Leibniz no se opone a que se llame 'uno' al arco iris o a otros fenómenos y piensa que se pueden contar, etc., pero no por ello alcanzan realidad metafísica. Inmediatamente después introduce una distinción entre materia segunda y primera.

La materia segunda

es la multitud de sustancias cuya masa es la del cuerpo entero (ibid.).

Y agrega:

puede decirse que esas sustancias son partes de esa materia (ibid.).

Pone como ejemplo nuestro cuerpo del que dice que tiene partes y es la materia; el alma es la forma y juntos forman la sustancia corporal. No aclara cómo la multitud de sustancias puede formar una masa ni es claro si esa masa es la que calificó antes como mero fenómeno o apariencia bien fundada. Leibniz confía en que el ejemplo de los humanos basta para disipar toda duda al respecto. Sin embargo, sigue sin responder a la cuestión de Arnauld, a saber, ¿cómo puede ser que lo que es múltiple se vuelva uno? Leibniz piensa que la dificultad puede resolverse si se tiene una tesis adecuada del todo y las partes:

Las partes pueden constituir un todo, haya o no haya verdadera unidad. Es cierto que el todo que tiene una verdadera unidad puede, en rigor, seguir siendo el mismo individuo. Aunque pierda o gane partes... (G. II, 119).

No es claro entonces cuándo un todo constituye una unidad verdadera y cuándo no basta que sea un todo para calificar como una unidad metafísica. Leibniz se conforma con repetir que nosotros experimentamos en nosotros mismos la unidad aun cuando perdemos y ganamos partes (sustancias, dijo más arriba). 
Finalmente, la materia primera la caracteriza como la potencia pasiva primitiva y dice que esa materia

no seria extensa ni divisible aun cuando constituya el principio de la divisibilidad o de lo que de ella aparece en la sustancia. (G. II, 120).

Reconstruyamos entonces la respuesta ${ }^{12}$ de Leibniz a Arnauld:

La materia no es plura entia sin más; como materia prima es unum ens y es esencial a la sustancia. La materia como materia segunda es un todo con unidad que puede perder o ganar partes. Leibniz no explica esto, es decir, cómo se da la unidad y se preserva a través de la pérdida y ganancia de partes. En vez de explicarlo, Leibniz pone el ejemplo de los seres humanos como si fuese bastante. En consecuencia, la materia segunda parece ser a la vez plura entia y unum ens. Es decir, la materia segunda parece ser una abstracción a partir de la multitud de sustancias que forman el cuerpo (humano) las cuales son ellas mismas unum ens y la totalidad así abstraída permanece una y la misma a pesar de la ganancia o pérdida de las partes (es decir de las sustancias que forman la masa o cuerpo).

Leibniz parece querer aquí dos tipos de unidad, la de las sustancias individuales que componen el todo y la del todo así compuesto que puede perder sustancias individuales o partes y sustituirlas por otras sin dejar de ser uno y el mismo todo. Esta concepción deja pendientes un sinnúmero de cuestiones que Leibniz rehusa contestar y se conforma con poner el ejemplo de nosotros mismos, es decir, de nuestros cuerpos.

Finalmente, declara a la materia como masa un mero agregado sin ninguna realidad -al parecer no califica tampoco para ser plura entiay la califica con un mero fenómeno, excrecencia o apariencia que tiene unidad por convención o de algún tipo metafísicamente sin importancia.

Permanece la duda acerca de si esta masa resulta de la multitud de sustancias que forman la sustancia corporal. En todo caso, Leibniz no explica cómo resulta la masa de la multitud o agregado de sustancias ni tampoco cómo las sustancias individuales que componen la sustancia material son ellas mismas materiales o corporales.

¿Puede ser acaso que la masa sea un fenómeno que producen en nosotros las agrupaciones de sustancias individuales pero que no existe como masa? En todo caso con esta interpretación no se puede resolver de ninguna manera el problema metafísico de la unidad de las sustancias materiales. 
3.3 Leibniz no ofrece una respuesta explicativa o elucidatoria a la objeción de Arnauld. Arnauld aduce sobre bases a priori que la unidad exige pluralidad y por lo tanto la pluralidad de los cuerpos queda irreducida.

La respuesta de Leibniz ${ }^{13}$ insiste en proponer un ejemplo: nosotros somos uno en virtud de tener un alma y sin embargo estamos compuestos de múitiples órganos y de partes que

están llenas, sin duda, de una infinidad de otras sustancias corpóreas dotadas de sus propias entelequias (ibid.).

Tenemos entonces, según se dijo a propósito de la materia segunda, unidad en lo alto, provista por el alma, y unidad en el extremo opuesto, constituida por la infinidad de sustancias corpóreas. En vez de explicación de cómo se conllevan estas dos unidades solamente nos ofrece un ejemplo, nosotros. ${ }^{14}$

3.4 Leibniz rechaza ${ }^{15}$ la dicotomía de Arnauld en sustancias o espirituales o corporales y formula la hipótesis de otros tipos de sustancias. Sin embargo, el vicio se vuelve a repetir: Leibniz no aclara el sentido de la unidad de esos otros tipos de sustancia. Por el contrario, parece creer que el siguiente paso consiste en que los científicos como Malpighi descubran esos otros tipos de sustancia. Pero la cuestión es si esos científicos van a descubrir auténticos individuos o solamente agregados de individuos que producen en nosotros la ilusión de unidad sin tenerla en realidad.

3.5 Leibniz afirma ${ }^{16}$ que en el caso del gusano de seda su alma puede estar con sus cenizas y deja sin aclarar la fuerza de este "puede". Sin embargo, se confiesa ignorante respecto a si las cenizas pueden generar otros gusanos. No dice si esta información adicional se obtendrá en el futuro y si será a priori o no.

3.6 Leibniz se auxilia con testimonios de científicos como Leewenhoeck y Swammerdam y dice ${ }^{17}$ que su juicio vale más al determinar si de las cenizas del cordero se pueden generar otros corderos. Leibniz ad-

19 Ibid., p. 120.

14 Dicho en otra forma aún: introducir otras sustancias como componentes de la sustancia material no resuelve nada. Lejos de resolver el problema lo agrava pues Leibniz no ha elucidado la unidad de esas sustancias componentes sino que la elimina con su idea de la simplicidad. Sobre esto véase el artículo citado antes en la nota 2.

15 Ibid., pp. 120-122.

16 Ibid., p. 122.

17 Ibid., pp. 122-124. 
mite que su tesis de la transformación va más allá de lo que esos cientificos admiten y recurre a la persuasión invitándonos a ver la muerte como un continuo en el que vamos de casos de cambios más o menos leves a metamorfosis, a caso de muerte clínica y llegamos a casos de muerte final. Es un mismo espectro con diferencias de grado y por lo tanto las cenizas $\mathbf{u}$ otra masa puede tener cuerpos organizados (G. II, 123). De nueva cuenta, ¿es éste un "puede" lógico o empírico; si es uno u otro, ¿cómo puede Leibniz establecerlo?

Alli mismo enfatiza Leibniz su tesis de que el tipo de sustancia corresponde al tipo de ley que rige su comportamiento (G. II, 124). En esa conexión dice que las sustancias espirituales no permiten cambio o revolución y por ello requieren de memoria o conciencia o reflexión, mientras que en las sustancias brutas basta que

sigan siendo simplemente el mismo individuo, en riguroso sentido metafísico, aunque estén sujetas a todos los cambios imaginables, puesto que no tienen conciencia o reflexión (ibid., p. 125).

Leibniz acepta múltiples tipos de sustancias; no dice que unas sean primarias frente a otras. Solamente afirma un pluralismo sustancial, con ciertas exigencias a priori como son la de tener unidad verdadera. Pero aun esas exigencias parecen poder satisfacerse de varias maneras y Leibniz no puede ofrecer una imagen coherente y razonablemente completa.

Resumamos entonces la concepción de las sustancias materiales individuales que presenta Leibniz al final de su Correspondencia con Arnauld.

La materia entendida como masa extensa es calificada como mera apariencia, resultado o subproducto de lo que es realmente.

La materia primera es la potencia pasiva primitiva que requiere toda alma, permanece siempre la misma y es el principio de la extensión y la divisibilidad en todo cuerpo.

Ninguna de estas dos concepciones de materia basta o es suficiente para que podamos tener una sustancia material individual, aun cuando la concepción de materia primera constituye un ingrediente necesario de la sustancia material. (Leibniz no nos dice cómo compone o se integra la materia primera con la sustancia material.)

Queda entonces la materia segunda, la cual es un agregado de sustancias, las cuales están unidas bajo una forma sustancial o alma. La tesis del todo y las partes ayuda a entender cómo una sustancia puede perder o ganar partes y, sin embargo, seguir siendo la misma. Esto es, ayuda a mantener un concepto de mismidad que no recurre a la noción de sustrato ni tampoco a la noción de composición. 
Pero como no siempre un todo es una sustancia, resulta que esa noción de todo debe suplementarse con otra u otras para poder llegar al concepto de sustancia. Como Leibniz no dice nada a este respecto, enumeraré una serie de posibilidades especulativas acerca de cómo, dadas las nociones que ha introducido Leibniz, puede lograrse la unidad de la sustancia material.

Si el alma es la forma sustancial, entonces ella deberá cumplir la tarea de unir la diversidad. He aquí algunas posibilidades:

i) Que la unidad se logre a través de la materia prima del alma que es el principio de la extensión de la sustancia corporal.

ii) Que el alma exprese a las sustancias corporales que componen la sustancia material y por medio de esa expresión las unifique.

iii) Que el alma sea solamente la conjunción coordinadora de todas y cada una de las formas sustanciales que unifican cada una de esas sustancias que componen la sustancia material qua materia segunda. De esta manera se resolvería la pérplejidad que se suscitó con la tesis de unidad por arriba y por abajo (cfr. 3.3).

iv) Que el conjunto de sustancias que componen la sustancia material qua materia segunda formen una unidad a través de las relaciones de expresión y es esa unidad resultante la que se denomina alma.

Creo que ninguna de las respuestas anteriores es satisfactoria porque cada una de ellas deja fuera de la unidad algo importante. Veamos:

(i) No explica la relación entre la materia primera del alma y la materia (o materias, primarias o secundarias) de las sustancias componentes. No es posible entender cómo esa materia primera que permanece siempre la misma da lugar a la materia segunda que varía constantemente. Menos aún se puede entender que esa materia primera vuelva una a varias sustancias que parecen estar completas ellas mismas. Es decir, si son sustancias las que componen la sustancia material debe cada una tener su alma, su materia primera y su materia segunda y por aquí parece que amenaza un regressus vicioso.

(ii) Es la tesis que aparece expuesta en la CA, G, II, p. 58 cuando Leibniz dice:

El alma, sin embargo, no deja de ser la forma de su cuerpo, porque expresa los fenómenos de todos los demás cuerpos según la relación que ellos tienen con el suyo.

Pero si bien la expresión podría explicar cómo el alma informa a la materia (segunda) esto no basta para explicar sino más bien presupone la unidad de cada una de las sustancias que intervienen en la relación de expresión. Es decir, esa materia está compuesta a su vez de sustancias 
ya unificadas y no sabemos cómo se constituye la unidad de cada una de esas sustancias. Y si no entendemos esto no entenderemos cómo se logra la unidad mayor de la sustancia material. No entenderemos, por ejemplo, si al formar la sustancia material, las sustancias componentes pierden su individualidad. Creo, en suma, que la tesis de la expresión presupone las unidades sustanciales y no puede contribuir a su constitución.

(iii) Modifica el esquema forma sustancial-materia pues ahora el alma es solamente la conjunción de muchas almas y todavía no sabemos cómo es que todas esas almas forman una sustancia. Es decir, parece que estaríamos hablando de algo así como una construcción lógica y esta idea se opone a la idea de forma sustancial la cual implica la noción de algo "sobre y por encima de aquéllo que lo constituye o compone".

(iv) También modifica el esquema dual de forma sustancial-materia pero reduciendo esta vez la primera a la segunda. También aquf nos quedamos con una diversidad de materias de las cuales se supone emerge una sola, pero esta unidad no tiene justificación. No es claro tampoco que la materia pueda ella sola expresar, pues la expresión tiene que ver con la representación y ésta es una propiedad de las mentes o almas.

\section{III}

Lejos de encontrarnos con una respuesta clara y coherente a las preguntas que formulamos al comienzo de este trabajo - y con ella a las objeciones que le presenta Arnauld- encontramos que las respuestas de Leibniz suscitan otras preguntas cada vez más complejas.

Por ningún lado aparece una respuesta clara a la cuestión de la unidad de las sustancias materiales. ${ }^{18}$ Calificarlas de meros fenómenos divierte la cuestión metafísica de su unidad y distinguir entre materia primera y segunda contrae dificultades adicionales para las cuales tampoco hay solución. En particular, no hay respuesta a la cuestión de cómo la materia segunda se unifica (¿con la materia primera?, de la cual dice Leibniz que resulta).

La tesis del todo y las partes no está desarrollada pero creo que tiende a resolver el problema de la unidad y de la identidad de esa unidad a través del tiempo. Posiblemente también se relacione la tesis del todo y la parte con la tesis de la ley que regula la complejidad de la

18 ¿Es acaso porque no puede explicar la unidad de las sustancias materiales con el modelo de la simplicidad que Leibniz afirma consoladoramente que:

es suficiente que las sustancias brutas sigan siendo simplemente el mismo individuo, en riguroso sentido metafísico, aunque estén sujetas a todos los cambios imaginables, puesto que no tienen conciencia o reflexión (G. II, 125). 
sustancia. Y ambas tesis pueden estar relacionadas a su vez con la tesis de la expresión.

No es claro, sin embargo, la conexión con la tesis de la unidad de las sustancias espirituales en términos de la idea de simplicidad. No es clara, en particular, la función que cumpliría la tesis de la expresión. A primera vista no parece que lo simple pueda expresar la complejidad de la multiplicidad de sustancias, menos aún unificar — hacer simplelo diverso.

Finalmente, está la tesis de la noción completa o concepto de sustancia y su conexión con las tesis mencionadas anteriormente. $Y$ esto se relaciona con la cuestión epistemológica de la determinación a priori de las mismas y de su carácter determinable a posteriori. Pero en estos puntos Leibniz permanece callado en estos escritos.

En otro lugar ${ }^{19}$ he tocado estos puntos pero es indudable que se necesita investigarlos con mayor detalle y claridad.

Para concluir, expresaré una duda fundamental. Hacia el final de la carta comentada del 9 de octubre de 1687 (G. II, 126) Leibniz reitera que la sustancia material qua masa divisible es mero fenómeno y qua sustancia

tiene una verdadera unidad, en riguroso sentido metafísico y que es indivisible, inengendrable e incorruptible; que toda materia debe estar llena de sustancias animadas o por lo menos vivas...

Es decir, Leibniz reitera la unidad ${ }^{20}$ y la diversidad de las sustancias componentes. ¿Es acaso que no puede decidirse a aceptar la materia como sustancia sin más? ¿Es por ello que en vez de conceder que la forma sustancial confiere unidad a una porción de materia prefiere decir que la materia en realidad es un conjunto de sustancias animadas? ¿Padece Leibniz el horror materiae o solamente finge padecerlo?

18 Cfr. "Sustancias y especies en los Nouveaux Essais." En: Didnoia 1982, UNAMFCE, México, pp. 129-142.

20 Pero entonces, ¿la unidad es algo mental solamente? $O$ acaso Leibniz es un realista extremo que nos dice: "la sustancia requiere unidad pero no sabemos cómo se logra ni lo que debemos conocer para llegar a conocerla". 